\title{
DESARROLLO DE LAS HABILIDADES SOCIALES DE ESTUDIANTES QUE INGRESAN A LA UNIVERSIDAD ${ }^{1}$
}

\author{
Emilio RODRIGUEZ-MACAYO² \\ Ruben VIDAL-ESPINOZA ${ }^{3}$ \\ Marco COSSIO-BOLAÑOS ${ }^{4}$
}

\begin{abstract}
${ }^{1}$ Este Artículo es producto de una investigación realizada por el autor principal auspiciada por la Dirección de Investigación y Postgrado (DIPOS) de la Universidad Católica Silva Henríquez (UCSH). Código S1804ERM. Denominado: "Nivel de desarrollo de las habilidades sociales en estudiantes de la Facultad de Educación cohorte de ingreso 2018 de la UCSH”. Elaborado en el segundo semestre académico 2017 y con plazo de finalización diciembre 2018.
\end{abstract}

${ }^{2}$ Universidad Católica Silva Henriquez. Santiago-Chile. ${ }^{2}$ Profesor de Educación Especial. Universidad de Salamanca. España. Máster en integración social de personas con discapacidad. Universidad de Salamanca. España. Académico e Investigador de la Carrera de Educación Diferencial de la Universidad Católica Silva Henríquez, Santiago -Chile. Académico Colaborador del Programa de Magister en Educación Mención Gestión Inclusiva Universidad Santo Tomás Sede Talca - Chile. Línea de Desarrollo Discapacidad Intelectual. Líneas de Investigación: Inclusión educativa. Procesos de transición a la vida adulta de persona en situación de discapacidad. Desarrollo de habilidades sociales. Miembro del Núcleo de Investigación y Desarrollo Interuniversitario de Chile y Macroproyecto Iberoamericano Pedagógico e Investigativo Curricular, Didáctico y Evaluativo de REDIPE - CHILE. Investigador Colaborador Grupo de Investigación Reconocido "Inclusión Educativa” Universidad de Salamanca- España. N ORCID 0000-0002-1321-2382. Membresía Red Iberoamericana de Pedagogía (REDIPE). erodriguez@ucsh.cl.

${ }^{3}$ Universidad Católica Silva Henriquez. Santiago-Chile

Profesor de Educación Especial y Diferenciada y Magister en Educación Especial de la Pontificia Universidad Católica de Chile. Académico e Investigador de la Carrera de Educación Diferencial de la Universidad Católica Silva Henríquez, Santiago -Chile. Académico Colaborador del Programa de Magister en Educación con Mención en Currículo de la Universidad Católica del Maule Talca -. Línea de Desarrollo Discapacidad Intelectual. Líneas de Investigación: Uso del conocimiento científico. Desarrollo de habilidades sociales. Miembro del Núcleo de Investigación y Desarrollo Interuniversitarios de Chile y Macroproyecto Iberoamericano Pedagógico e Investigativo Curricular, Didáctico y Evaluativo de REDIPE - CHILE. N Registro ORCID 0000-0002-8593-5249. rvidale@,ucsh.cl.

${ }^{4}$ Universidad Católica del Maule. Talca. Chile

Profesor de Educación Física. Universidad Nacional de San Marco. Perú. Doctor en Ciencias del Deporte. Universidad Estadual de Campinas. Brasil. Postdoctorado Profesor de Educación Física. Universidad Nacional de San Marco. Perú. Doctor en Ciencias del Deporte. Universidad Estadual de Campinas. Brasil. Postdoctorado Biomecánica del Movimiento Humano. Universidad Estadual Campinas. Brasil. Coordinador y Docente del Doctorado de Ciencias de la Actividad Física de la Facultad de Educación. Universidad Católica del Maule. Talca-Chile. Docente y Tutor de Tesis del Programa de Magister de Ciencias de la Actividad Física. Facultad de Educación. Universidad Católica del Maule. Talca-Chile. Es autor de un número significativo de artículos en revistas indexadas de alto impacto. Liderados procesos de investigación en el área de Educación. Director del Nucleo de Investigación del Desarrollo Biológico y Humano. Facultad de Educación. Universidad Católica del Maule. Talca-Chile. mcossio@ucm.cl

Fecha de recepción del artículo: Dicienbre 1918

Fecha de evaluación: Marzo 2019 


\title{
Resumen
}

El artículo da cuenta de resultados de una investigación cuyo objetivo fue determinar el nivel de desarrollo de las habilidades sociales de estudiantes que ingresan a la universidad. La metodología es de tipo cuantitativo, descriptivo, transeccional no experimental. Se encuesta a 446 estudiantes de 9 carreras de la Facultad de Educación. Entre sus resultados destaca que no existen diferencias significativas entre las 6 dimensiones del cuestionario al comparar por género, edad y vía de ingreso a la universidad. A modo de conclusión es posible señalar que el instrumento es válido y confiable pudiendo ser aplicado en contextos académicos.

Palabras Clave: Desarrollo, habilidades, sociales, estudiantes, universitarios

\begin{abstract}
The article reports the results of a research whose objective was to determine the level of development of the social skills of students entering the university. The methodology is quantitative, descriptive, non-experimental transectional. 446 students of 9 careers of the Faculty of Education are surveyed. Among its results it stands out that there are no significant differences between the 6 dimensions of the questionnaire when comparing by gender, age and university entry route. By way of conclusion it is possible to point out that the instrument is valid and reliable and can be applied in academic contexts.
\end{abstract}

Keywords: Development, skills, social, students, university

\section{Résumé}

L'article présente les résultats d'une recherche dont l'objectif était de déterminer le niveau de développement des compétences sociales des étudiants entrant à l'université. La méthodologie est quantitative, descriptive, transectionnelle non expérimentale. L'enquête porte sur 446 étudiants de 9 carrières de la Faculté d'éducation. Parmi ses résultats, il ressort qu'il n'y a pas de différence significative entre les 6 dimensions du questionnaire lors de la comparaison par sexe, âge et voie d'entrée à l'université. En guise de conclusion, 
il est possible de souligner que l'instrument est valide et fiable et peut être appliqué dans des contextes académiques.

Mots-clés: Développement, compétences, social, étudiants, université

\section{Introducción.}

El desarrollo de las habilidades sociales (HHSS) de estudiantes universitarios es un problema general, de alta preocupación de los docentes y de la investigación académica, que se genera en las Instituciones de Educación Superior (IES), tanto a nivel nacional como a nivel internacional y que se encuentra a la vez altamente evidenciado y se ha descrito ampliamente de aquello en el ámbito de la psicología y de la educación, (Miranda -Zapata, Riquelme-Mella, Cifuentes-Cid, y Riquelme-Bravo, 2014). Se asume, al respecto que, el desarrollo de las HHSS satisfactorias permitiría a los estudiantes universitarios, próximo a su egreso, desempeñarse de manera exitosa y robusta en diferentes contextos, así como en los vínculos y relaciones afectivas cercanas, como en el ámbito académico, laboral y en la vida profesional. (Morán, y Olaz, 2014).

Respecto de lo anterior, es posible señalar que, en la actualidad, la importancia del nivel del desarrollo de la HHSS de estudiantes universitarios en el plano del funcionamiento interpersonal es un tema que está ampliamente documentado (Hidalgo, y Abarca, 1990; Gallego-Villa, 2008; Campo-Ternera, y Martínez de la Brava, 2009; Ruíz-Arias, y Jaramillo-Campillo, 2010; Ordaz-Hernández, 2013; Bueno-Moreno, Durán-Segura, y Garrido-Torres, 2013; Lisette-Segura, y López, 2014; Sánchez - Teruel, Robles - Bello, y González - Cabrera, 2015; Rodriguez-Macayo y Vidal-Espinoza, 2018).

Del anterior planteamiento es posible señalar que el desarrollo de la HHSS está en estrecha relación a la base del éxito laboral.

Este problema se convierte en un objeto de investigación cuando se articulan preguntas sobre las características de aquello, de sus relaciones con otros y en el otro (en lo cotidiano y en lo diario, en la convivencia y en las relaciones humanas en todo lugar y momento). Sobre la base de estas preguntas y sus respuestas, es posible elaborar un marco conceptual teórico y al mismo tiempo elaborar una estrategia metodológica continua para favorecer el desarrollo de la HHSS de estudiantes que ingresan a la universidad. 
Finalmente, señalar que esta investigación, está centrada desde la mirada de una dimensión formativa de los estudiantes, buscando y encontrando respuestas para favorecer planes de formación inicial en la formación de profesionales noveles, acorde a la misión institucional de nuestra universidad que encarna el carisma salesiano al servicio de los sectores prioritarios de la sociedad.

\section{Fundamentación}

Las HHSS hacen mención a las destrezas específicas necesarias en toda persona que le permiten enfrentar con éxito situaciones interacciónales. A la base de este planteamiento se encuentra la inteligencia interpersonal definida esta como la destreza para comprender a los otros, motivarlos y cooperar con ellos. Se está por lo tanto ante capacidades adquiridas que se pueden enseñar, aprender y practicar (Gallego-Villa, 2008).

No obstante, lo anterior pareciera que no existe una única definición consensuada, más bien lo que existen son características fundamentales que se reiteran en cada una de ellas, estas son: Conductas manifiestas; es decir, son un conjunto de estrategias y capacidades de actuación aprendidas y que se manifiestan en situaciones de relaciones interpersonal, incluyendo comportamientos verbales y no verbales, específicos y discretos. Están dirigidas a la obtención de reforzamientos sociales tanto externos como internos o personales (autorrefuerzo, autoestima). Implican una interacción recíproca. Están determinadas por el contexto social, cultural y la situación concreta y específica en que tiene lugar. Las HHSS se organizan en distintos niveles de complejidad, los cuales mantienen una cierta jerarquía, cuyas estructuras se desarrollan desde un nivel molar (por ejemplo: habilidades de interacción heterosocial), hasta llegar a uno molecular (por ejemplo: mantener contacto ocular), habiendo pasado por niveles intermedios (por ejemplo: decir "no"). Como todo tipo de conducta, se encuentran muy mediatizadas por las ideas y creencias respecto a la situación y a la actuación propia de los demás. Tanto los déficits como los excesos de la conducta de interacción personal pueden ser especificados y operacionalizados con el propósito de intervenir sobre ellos. (GallegoVilla, 2008)

Al ser un concepto de tipo descriptivo, es importante caracterizar el desempeño social del individuo partiendo de ciertos elementos, que según Riso (1988) y Caballo (1993) serían 
los componentes comportamentales manifiestos (moleculares y molares). Los mediadores cognitivo-afectivos (pensamientos y sentimientos involucrados en la decodificación de las demandas interpersonales de la situación, en la decisión sobre el desempeño requerido para dicha situación, y en la elaboración y auto monitoreo de ese desempeño). Los Componentes fisiológicos (procesos sensoriales y de regulación del control autónomo), y finalmente los componentes situacionales, referidas al contexto ambiental.

Respecto de lo anterior, es necesario indicar, que en el mercado actual competitivo se manifiesta la necesidad relativa a la formación de profesionales en el desarrollo de las HHSS (Becker, 1973; Cejudo, 2006), que les permitan sostener vínculos sanos consigo mismo y con los demás, es decir, un profesional competitivo que responda a las demandas del medio, del contexto, orientados al trabajo de interacción colectiva más que individual (Ruiz-Arias y Jaramillo-Campillo, 2010). Al respecto estos autores sostienen que:

\footnotetext{
Una persona con bajo nivel de desarrollo de las HHSS, a menudo, no sabe expresar sus Ideas, reprime sus emociones y que a la vez le es difícil solicitar favores, y/o buscar apoyo para realizar sus actividades por sencillas que ellas sean, para resolver situaciones que puedan ocurrir. (p. 55).
}

Según se indica anteriormente, podría desprenderse que una persona con un adecuado nivel de desarrollo de las HHSS podría sostenerse con éxito en un puesto de trabajo en un empleo competitivo y además desarrollarse óptimamente en aquello, dado que a la base de la vida del trabajo constituye una condición nuclear, sus relaciones podrían ser satisfactorias y duraderas y su producción podría ser más productiva.

Las HHSS, en cuanto a su origen se remontan a Salter (1947), según Campo-Ternera y Martínez-De Bravo (2009), ellas constituyen un constructo con dificultad para precisar, es decir, algo que se sabe y se ha estudiado mucho de aquello y que sin embargo es difícil de precisar.

En esta sentido, es posible precisar, que las HHSS constituyen un conjunto de complejos comportamientos interpersonales acompañados a factores de aprendizajes (Michelson y Wood, 1980; Belleck y Morrison, 1982; Abarca-Hidalgo, 1990) constituye la capacidad de la ejecución de conductas de intercambio con resultados favorables que permitan interactuar con sus pares y en entorno de manera socialmente aceptable: Saludar, sonreír, 
hacer y pedir favores, iniciar y mantener una conversación, evitar y manejar una agresión. Son comportamientos o pensamientos absolutamente instruméntale al desarrollo de la vida y constituyen condiciones transversales para resolver conflictos en situaciones cotidianas. Al respecto ellas tienen tres componentes: El logro de los objetivos cotidianos, mejorar situaciones de contexto y mantener una autoestima lo que implica manejarse desde una dimensión conductual, una dimensión personal y una dimensión situacional. Acorde a lo anterior, Ruíz-Arias y Jaramillo-Campillo (2010), señalan que las HHSS constituyen un conjunto complejo de comportamiento interpersonal que dependen, en gran medida, de factores de aprendizaje y estos autores las entienden como las capacidades para organizar cogniciones y conductas en un trayecto de acciones dirigido hacia el logro de metas sociales. En atención al problema se debe tener en cuenta los contextos enmarcados en los aspectos sociales, culturales y familiares desde una mirada de los procesos de la comunicación.

Caballo $(1993,2007)$ sostiene es muy probable que frente a definiciones de las HHSS no se llegue a acuerdo en ello. Meichenbaum, Bluter y Gruson (1981) por su parte sustentan que se debe tener en cuenta los contextos enmarcados en lo social, lo cultural, lo familiar a la hora de intencionar el desarrollo de las HHSS.

La revisión del estado del arte de la evidencia investigativa permite señalar que autores tales como Iruarrizaga, Gómez-Segura, Criado, Zuazo y Sastre (1997) y con CamposTernera y Martínez De Biava (2009) consideran que una de las definiciones más completa de las HHSS es la señalada por Caballo (1986, 2007), quien las define como:

El conjunto de conductas emitidas por un individuo en un contexto interpersonal que expresa los sentimientos, actitudes, deseos, opiniones o derechos de ese individuo de un modo adecuado a la situación inmediata, respetando esas conductas en los demás, y que generalmente resuelve los problemas inmediatos de la situación mientras minimiza probabilidad de futuros problemas (p. 40).

Otra definición importante a señalar encontrada en la revisión bibliográfica es la enunciada por Monjas (2007), para este investigador las HHSS constituyen un "un numeroso y variado conjunto de conductas que se ponen en juego en situaciones de 
interacción social, es decir, en situaciones en las que hay que relacionarse con otras personas" (p. 40).

Sobre la base de las consideraciones anteriores (Buela-Casal, Caballo y Sierra, 1996; Caballo 2000; Campos-Ternera y Martínez De Biava, 2009) plantean que las HHSS se manifiestan en entornos y contextos específico y en determinadas personas, entre amigos, entre personas de diferentes sexo, entre la familia y sus relaciones más estrecha (padres, madres, hermanos y otros), en el trabajo (autoridad, compañeros del trabajo del mismo sexo y otros), en el jardín infantil, en la escuela, en liceo, en la universidad, en los lugares de diversión y de ocio.

\section{Metodología}

La investigación se realizó bajo el paradigma positivista. Corriente que asume que es posible desarrollar una investigación libre de valores. En donde la realidad es única y puede ser fragmentada para su análisis y las partes pueden ser manipuladas independientemente. La investigación positivista tiene un enfoque metodológico predominantemente cuantitativo. Esta corriente de pensamiento no admite como válidos otros conocimientos sino los que proceden de las ciencias empíricas. Para Kolakowski (1988) el positivismo es un conjunto de reglamentaciones que rigen el saber humano y que tiende a reservar el nombre de ciencia a las operaciones observables.

El presente estudio es de tipo cuantitativo pudiendo ser clasificado como una investigación básica con dos propósitos. En el primer caso con aumentar los conocimientos y saberes frente al nivel de desarrollo de las HHSS de los estudiantes de ingreso cohorte 2018 a la Facultad de Educacion de la USCH y en un segundo caso con generar estrategias metodológicas de apoyo al desarrollo de las HHSS.

En cuanto al diseño este es de tipo estudio descriptivo- survey, transversal-transeccional, no experimental.

La población se constituyó por todos los estudiantes de ingreso cohorte 2018 de nueve

(9) Carreras de Pregrado pertenecientes a la Facultad de Educacion la USCH.

La determinación específica del número de elementos a estudiar (unidades de análisis), se calculó a un nivel de confianza de un 95\% (1.96) con un margen de error muestral de un $5 \%(0.05)$. La muestra es de tipo probabilística, lo que indica que todos los estudiantes 
de la población tuvieron la misma probabilidad de formar parte de ella. Su selección se efectuó mediante procedimientos matemáticos. (ver tabla 1)

Tabla 1. Distribución de la Muestra

\begin{tabular}{rcc} 
Carreras & $\mathrm{n}$ & $\%$ \\
\hline Pedagogía en Educacion Diferencial & 92 & 20,6 \\
Educación Parvularia & 72 & 16,1 \\
Pedagogía en Educación Técnica & 11 & 2,5 \\
Pedagogía en Educación Artística & 35 & 7,8 \\
Pedagogía en Matemática e & & \\
Informática & 28 & 6,5 \\
Pedagogía en Educación Básica Vesp. & 30 & 6,7 \\
Pedagogía en Educación Básica & & \\
Diurna & 47 & 10,5 \\
Pedagogía en Educación Física & 93 & 21,9 \\
Pedagogía en Inglés & 38 & 8,5 \\
& & \\
\hline Total & 446 & 100,00
\end{tabular}

Elaboración propia.

Se utilizó la técnica de la encuesta y para la recolección de los datos se aplicó un cuestionario de Escala (tipo) Likert.

Se aplica el cuestionario denominado "Cuestionario para evaluar el nivel de desarrollo de las HHSS de estudiantes universitarios" de Goldstein, Sprafkin, Gershaw y Klein (1989). Para tal efecto se solicitó autorización correspondiente a Martinez e editores.

El instrumento tiene una primera parte donde se rescatan datos de información sociodemográfica de los participantes. A continuación, el instrumento presenta seis dimensiones, a saber: HHSS básicas con ocho (8) indicadores (o reactivos). HHSS avanzadas con seis (6) indicadores. HHSS relacionadas con los sentimientos con siete (7) indicadores. HHSS alternativas a la agresión con ocho indicadores. HHSS para hacer frente al estrés con doce (12) indicadores. HHSS de planificación con ocho (8) indicadores.

Para su validez interna y externa se utilizó el método de validez de constructo. Se aplicó el análisis factorial exploratorio. La confiabilidad se determinó por medio de consistencia interna (Alpha de Cronbach). 
El porcentaje de la varianza explicó 41.4\%. La medida de Kaiser-Meyer-Olkin KMO fue de 0.906 y la prueba de esfericidad de Bartlet fue altamente significativa $\left(\mathrm{X}^{2}=11020.251\right.$, $\mathrm{gl}=1225, \mathrm{p}<0.001)$. Las cargas factoriales en las seis dimensiones y en las 50 preguntas oscilaron entre 0.42 a 0.72 y la fiabilidad en general evidenció un Alpha de Cronbach $(\mathrm{r}=0.92)$. Lo anterior nos permite decir que estamos frente a un cuestionario valido $\mathrm{y}$ confiable.

El instrumento se aplicó a todos los estudiantes seleccionados usando método tradicional, papel y lápiz contando con el tiempo requerido para aquello. Contestaron todos los estudiantes seleccionados y que asistieron el día de la aplicación firmando a su vez un consentimiento informado.

Una vez recolectados los datos proporcionados por el instrumento, se procedió al análisis estadístico respectivo. Se aplica estadígrafos, análisis de frecuencias, presentación de figuras y tablas con sus respectivos comentarios. Entre las medidas de tendencia central se aplican mediana, promedios y porcentajes. En cuanto a las medidas de dispersión se realiza mediante desviación estándar y análisis de varianza. Toda la información se procesó mediante paquete estadístico para las Ciencias Sociales (SSPS) Versión 20.

Según se destaca en la tabla 2 y en función de la variable género es posible señalar que en la categoría Femenino hay una presencia de 332 unidades de análisis, lo que supone un $74,4 \%$ por sobre la categoría masculina con una presencia de 114 lo que implica un 25,6 \%. En la función de la variable Edad es posible señalar que 342 estudiantes se ubican en una categoría de entre 17 y 21 años de edad lo que supone un 76,5\%. Le sigue la categoría entre 21.1 y 26 años con 63 estudiante que equivalen a 14,3. A partir de acá la frecuencia comienza a disminuir. Respecto a la variable Vía de Ingreso la mayor presencia de estudiantes se da en el Proceso de Selección Universitaria (PSU) con 385 estudiantes que suponen un 86,3\%. Llama la atención el ingreso por medio del Programa de Acompañamiento y Acceso efectivo a la Educación Superior (PACE) con 23 estudiantes correspondiendo a un 5,2\%. (ver tabla 2 ) 
Tabla 2: Características de los estudiantes estudiados en función del Género, Edad y Vía de ingreso a la Universidad. $(\mathrm{N}=446)$

\begin{tabular}{llrrr}
\hline & & fi & X & \multicolumn{1}{c}{$\%$} \\
\hline Género & & & & \\
& Masculino & 114 & 0,26 & 25,6 \\
& Femenino & 332 & 0,74 & 74,4 \\
Edad & & 446 & 1,00 & 100,0 \\
& & & & \\
& Entre 17 y 21 a & 342 & 0,76 & 76,5 \\
& Entre 21.1. y 26 a & 63 & 0,14 & 14,3 \\
& Entre 26.1 y 31 a & 22 & 0,05 & 4,9 \\
& Entre 31.1 y 36 a & 6 & 0,01 & 1,3 \\
& Más de 36.1 a & 13 & 0,03 & 2,9 \\
& & 446 & 1,00 & 100,0 \\
Vía ingreso & & & \\
& PSU & 385 & 0,86 & 86,3 \\
& Convalidación & 7 & 0,02 & 1,6 \\
Bachillerato & 10 & 0,02 & 2,2 \\
PACE & 23 & 0,05 & 5,2 \\
Otro & 21 & 0,05 & 4,7 \\
\hline & 446 & 1,00 & 100,0 \\
\hline
\end{tabular}

Elaboración Propia. Leyenda: PSU: Prueba de selección universitaria, PACE: Programa de Acompañamiento y Acceso Efectivo a la Educación Superior,

\section{Análisis de Resultados}

A continuación, se presenta el análisis de resultados, en donde en la Tabla 3, muestra el promedio y porcentaje obtenido en cada una de las Dimensiones que constituyen el cuestionario. Al respecto señalar que los mayores niveles de desarrollo de las HHSS se encuentran en aquellos que dicen relación con la alternativa a la agresión con un promedio de 4,2 lo que equivale a un nivel de desarrollo de un $84.15 \%$. Levemente inferior se encuentran la HHSS de planificación con un nivel de desarrollo de un 83.49\%. Bajo las anteriores se encuentran las HHSS relacionadas con sentimiento observando un promedio 
de 3,9 correspondiéndole un 77,66 \%. Llama la atención que todos los niveles de desarrollo de HHSS de los estudiantes de ingreso cohorte 2018 a la Facultad de Educacion de la USCH se encuentran por sobre un 76,6 \% en todas aquellas dimensiones propuestas en el cuestionario, lo que permite describir que habría un buen nivel de desarrollo de estas habilidades en estos estudiantes. (Ver tabla 3)

Tabla 3. Promedio y Porcentaje por Dimensiones de Estudio.

\begin{tabular}{lcc}
\hline & $\mathrm{X}$ & $\%$ \\
\hline HHSS básicas & 4,1 & 81,24 \\
HHSS avanzadas & 4,0 & 79,71 \\
$\begin{array}{l}\text { HHSS relacionadas con } \\
\text { sentimientos }\end{array}$ & 3,9 & 77,66 \\
$\begin{array}{l}\text { HHSS alternativas a la } \\
\text { agresión }\end{array}$ & 4,2 & 84,15 \\
$\begin{array}{l}\text { HHSS para hacer frente al } \\
\text { estrés }\end{array}$ & 4,0 & 79,80 \\
HHSS de planificación & 4,2 & 83,49 \\
\hline & 4,1 & 81,01 \\
\hline
\end{tabular}

Elaboración Propia. Leyenda: HHSS: Habilidades sociales.

Según se observa en la tabla 4 es posible señalar, a modo general, que hay levemente un mejor desempeño de las habilidades sociales en la categoría Femenino $(81.58 \%)$ por sobre la categoría masculino existiendo un $79.33 \%$. De la tabla se desprende que el nivel de desarrollo de habilidades sociales relacionadas con sentimiento en la categoría masculino es la más descendida con un 74,81\%, siendo las habilidades sociales de planificación con un mayor nivel de desarrollo con un $81.89 \%$. Llama la atención que los resultados o desempeños de la categoría masculino en el nivel de desarrollo señalado son las mismas que se aprecian en la categoría femenino. Por su parte, en la categoría femenino, los niveles de desarrollo más descendidos corresponden a aquellas relacionadas con sentimiento con un promedio de 3.9 equivalente a un $78.64 \%$. Aquellas que se vinculan con alternativas a la agresión presentan el mayor nivel de desarrollo con un 4,2 de promedio correspondiente al $84.47 \%$. (Ver tabla 4 ). 
Tabla 4 Niveles de desarrollo de Habilidades Sociales en cuanto al Género.

\begin{tabular}{lcccccc}
\hline & \multicolumn{2}{c}{ Masculino } & \multicolumn{2}{c}{ Femenino } & \multicolumn{2}{c}{ Total } \\
& $\mathrm{X}$ & $\%$ & $\mathrm{X}$ & $\%$ & $\mathrm{X}$ & $\%$ \\
\hline HHSS básicas & 3,9 & 78,57 & 4,1 & 82,16 & 4,1 & 81,24 \\
HHSS avanzadas & 3,9 & 78,36 & 4,0 & 80,17 & 4,0 & 79,71 \\
$\begin{array}{l}\text { HHSS relacionadas con } \\
\text { sentimientos }\end{array}$ & 3,7 & 74,81 & 3,9 & 78,64 & 3,9 & 77,66 \\
$\begin{array}{l}\text { HHSS alternativas a la } \\
\text { agresión }\end{array}$ & 4,2 & 83,20 & 4,2 & 84,47 & 4,2 & 84,15 \\
$\begin{array}{l}\text { HHSS para hacer frente al } \\
\text { estrés }\end{array}$ & 4,0 & 79,17 & 4,0 & 80,01 & 4,0 & 79,80 \\
HHSS de planificación & 4,1 & 81,89 & 4,2 & 84,04 & 4,2 & 83,49 \\
\hline & 4,0 & 79,33 & 4,1 & 81,58 & 4,1 & 81,01 \\
\hline
\end{tabular}

Elaboración Propia. Leyenda: HHSS: Habilidades sociales.

Entre la variable edad y sus diferentes distribuciones es posible señalar que el desarrollo relacionado con sentimientos se advierte como el más descendido con un promedio de 3,9 con un porcentaje $77.66 \%$, lo que refleja en las categorías entre 17 y 21 años, entre 21.1 y 26 años y más de 36.1 años. De mismo modo el mayor nivel de desarrollo de las habilidades sociales se observa en la dimensión relacionada con alternativa a la agresión obteniendo un promedio de 4.2 con un $84.15 \%$. Según lo descrito anteriormente es posible señalar que a nivel general todas las categorías de las dimensiones HHSS alternativas a la agresión y de planificación se encuentran por porcentajes sobre $82,50 \%$. De igual manera es posible indicar que estos estudiantes presentan un buen desarrollo de HHSS al ingreso a la universidad según la variable edad en sus diferentes categorías. (ver tabla 5) 
Tabla 5: Niveles de desarrollo de Habilidades Sociales en cuanto a la Edad.

\begin{tabular}{|c|c|c|c|c|c|c|c|c|c|c|c|c|}
\hline & \multicolumn{2}{|c|}{$\begin{array}{c}\text { Entre } \\
17 \text { y } 21\end{array}$} & \multicolumn{2}{|c|}{$\begin{array}{c}\text { Entre } \\
21.1 \text { y } 26\end{array}$} & \multicolumn{2}{|c|}{$\begin{array}{c}\text { Entre } \\
26.1 \text { a } 31\end{array}$} & \multicolumn{2}{|c|}{$\begin{array}{c}\text { Entre } \\
31.1 \text { y } 36\end{array}$} & \multicolumn{2}{|c|}{$\begin{array}{l}\text { Más de } \\
36.1\end{array}$} & \multicolumn{2}{|c|}{ Total } \\
\hline & $X$ & $\%$ & $X$ & $\%$ & $X$ & $\%$ & $\mathrm{X}$ & $\%$ & $\mathrm{X}$ & $\%$ & $\mathrm{X}$ & $\%$ \\
\hline HHSS básicas & 4,1 & 81,08 & 4,0 & 80,24 & 4,1 & 82,84 & 4,4 & 87,50 & 4,2 & 84,81 & 4,1 & 81,24 \\
\hline $\begin{array}{l}\text { HHSS } \\
\text { avanzadas }\end{array}$ & 4,0 & 79,67 & 4,0 & 79,21 & 4,0 & 79,70 & 4,2 & 84,44 & 4,1 & 81,03 & 4,0 & 79,71 \\
\hline $\begin{array}{l}\text { HHSS } \\
\text { relacionadas } \\
\text { con } \\
\text { sentimientos }\end{array}$ & 3,9 & 77,87 & 3,7 & 74,69 & 4,1 & 81,82 & 4,2 & 83,33 & 3,8 & 76,92 & 3,9 & 77,66 \\
\hline $\begin{array}{l}\text { HHSS } \\
\text { alternativas a la } \\
\text { agresión }\end{array}$ & 4,2 & 84,17 & 4,1 & 82,50 & 4,3 & 85,35 & 4,5 & 90,74 & 4,3 & 86,32 & 4,2 & 84,15 \\
\hline $\begin{array}{l}\text { HHSS para } \\
\text { hacer frente al } \\
\text { estrés }\end{array}$ & 4,0 & 79,64 & 4,1 & 81,01 & 4,0 & 80,91 & 3,9 & 78,06 & 3,9 & 77,05 & 4,0 & 79,80 \\
\hline $\begin{array}{l}\text { HHSS de } \\
\text { planificación }\end{array}$ & 4,2 & 83,34 & 4,1 & 82,98 & 4,2 & 83,52 & 4,4 & 88,75 & 4,4 & 87,50 & 4,2 & 83,49 \\
\hline & 4,0 & 80,96 & 4,0 & 80,10 & 4,1 & 82,36 & 4,3 & 85,47 & 4,1 & 82,27 & 4,1 & 81,01 \\
\hline
\end{tabular}

Elaboración Propia. Leyenda: HHSS: Habilidades sociales.

Respectivamente de la variable vía de ingreso y sus diferentes distribuciones es posible señalar que el nivel de desarrollo de las HHSS relacionadas con sentimientos se advierte como la más descendida en todos sus promedios. Del mismo modo es posible señalar que el mejor nivel de desarrollo lo encontramos en aquellas habilidades que hacen relación con alternativas a la agresión y a la planificación con porcentajes superiores al 79.75\%. Es atendible que los estudiantes que ingresan cohorte 2018 a la Facultad de Educación de esta universidad mediante vía proceso de selección universitaria (PSU) presentan niveles de desarrollo de habilidades sociales en el promedio general. (Ver tabla 6) 
Tabla 6: Niveles de desarrollo de Habilidades Sociales en cuanto a la Vía de Ingreso a la Universidad.

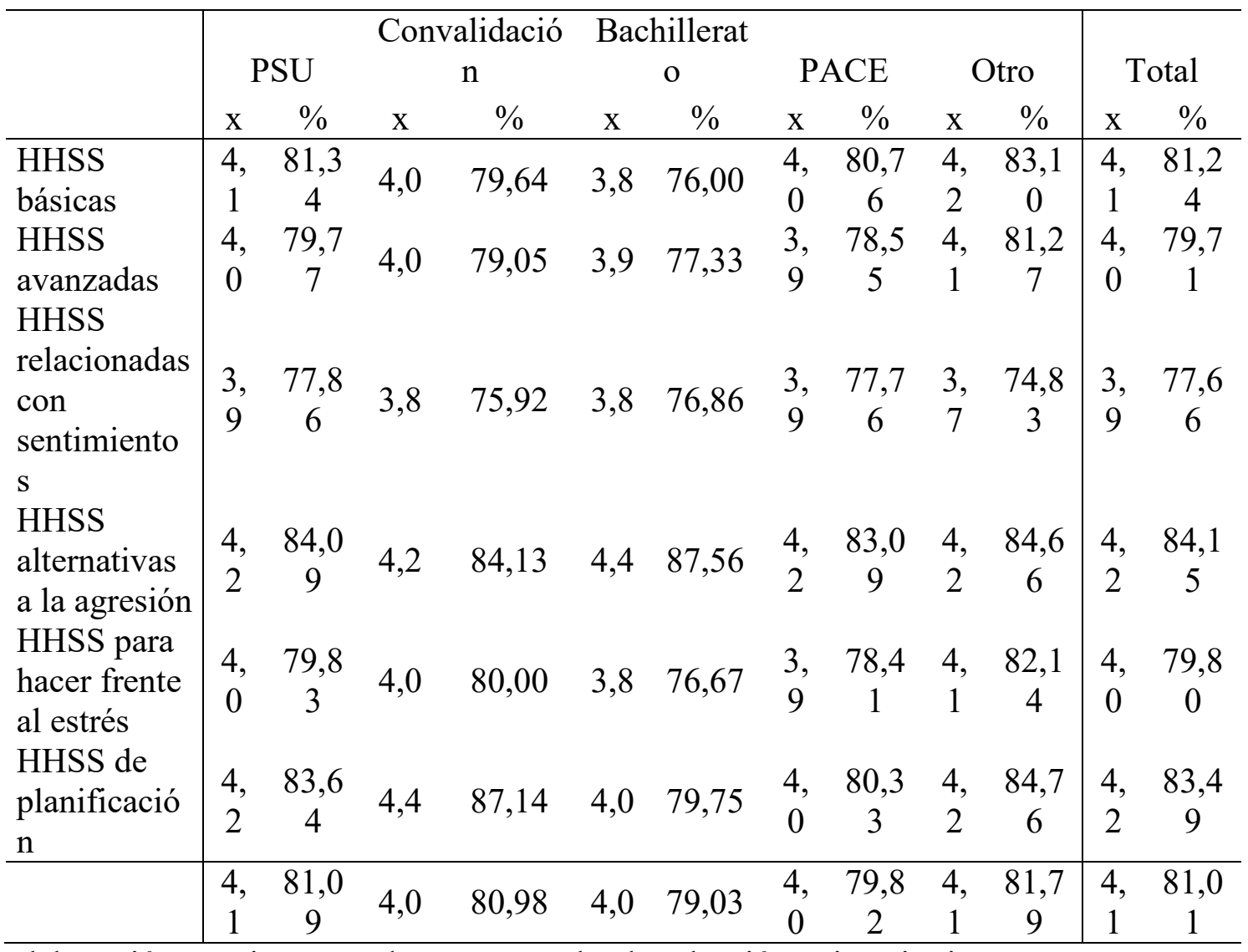

Elaboración Propia. Leyenda: PSU: Prueba de selección universitaria, PACE: Programa de Acompañamiento y Acceso Efectivo a la Educación Superior, HHSS: Habilidades sociales.

\section{Resultados}

El objetivo de este estudio buscó determinar el nivel de desarrollo de las habilidades sociales en estudiantes que ingresan por primera vez a la Facultad de Educación de una IES de de Santiago de Chile. Para tal efecto se aplicó una escala de HHSS con seis dimensiones y 50 preguntas. Entre los resultados obtenidos es posible señalar que todos estos estudiantes presentan un buen nivel de desarrollo de habilidades sociales en todas las dimensiones que propone el cuestionario al compararlo por género, edad y vía de ingreso. Lo anterior supone que no existen diferencias significativas entre los resultados de todos estos estudiantes universitarios en las seis dimensiones propuestas.

Estos resultados obtenidos son similares a otro estudio realizado en poblaciones de estudiantes universitarios (Miranda-Zapata et al 2014, Morán y Olaz, 2014). 
A modo de conclusión de este estudio cabe mencionar que el cuestionario utilizado es válido y confiable pudiendo ser utilizado en la evaluación del desarrollo de habilidades sociales de estudiantes en contextos universitarios.

\section{Referencias.}

ABARCA, N y HIDALGO. C. G. (1989). Evaluación psicométrica de habilidades sociales en jóvenes universitarios chilenos. Revista Análisis del Comportamiento, 4, 5-62

BECKER, G. (1983). El capital humano: un análisis teórico y empírico referido fundamentalmente a la educación. Madrid: Alianza

BELLACK, A. Y MORRISON, R. (1982). Interpersonal dysíunctíon, En A. J. Bellack, M. Hersen, y A.E .. Kazdin (Edl.), International handbook of behavior modification and therapy. Nueva York: Plenum Press

BUELA-CASAL, G., CABALLO, V. Y SIERRA, J. C. (1996). Manual de evaluación en psicología clínica y de la salud. Madrid: Siglo XXI.

BUENO-MORENO, M․, DURÁN-SEGURA, M. y GARRIDO-TORRES, M. (2013). Entrenamiento en HHSS en estudiantes universitarios de magisterio de la especialidad de educación primaria: un estudio preliminar. Sevilla, España. 31(1): 85-91. Recuperado de: www.apuntesdepsicologia.es/index.php/ revista/article/viewFile/301/287

CABALlO, V. (1986) Evaluación de las habilidades sociales. En Fernández R. y Carboles J.A. Evaluación conductual: metodología y aplicaciones. Madrid: Pirámide

CABALLO, V. (1993). La multidimensionalidad conductual de las Habilidades Sociales: propiedades psicométricas de una medida de autoinforme, la EMES-M. Psicología conductual, 1(2), 221-231.

CABALLO, V. (2007) Manual de evaluación y entrenamiento de las habilidades sociales. Madrid: Siglo Veintiuno Editores.

CAMPO-TERNERA, L. y MARTÍNEZ DE LA BRAVA, Y. (2009). HHSS de estudiantes de psicología de una universidad privada de la costa Caribe Colombia. Colombia. Revista Iberoamericana de Psicología Ciencia y Tecnología. 2 (1): 39- 51.

CEJUDO, R. (2006). Desarrollo humano y capacidades. Aplicaciones de la teoría de las capacidades de Amartya Sen a la educación. Revista española de Pedagogía (234), $365 \_380$.

COSSIO - BOLAÑOS, M. A. (2015). Métodos de investigación cuantitativa en ciencia de la educación. Talca, Chile. Texto de Apoyo a la Docencia: Universidad Católica del Maule

FERNÁNDEZ, M., BENÍTEZ, J., PICHARDO, M., FERNÁNDEZ, E. JUSTICIA, F., GARCÍA, T,... ALBA, G. (2010). Análisis factorial confirmatorio de las subescalas del 
pkbs-2 para la evaluación de las hhss y los problemas de conducta en educación infantil. España. Electronic Journal of Research in Educational Psychology. 8 (33): 1696 - 2095.

GALlEGO-VILLA, O. (2008). Descripción de las HHSS en estudiantes de psicología de una institución de educación superior. Bogotá, Colombia. Revista Iberoamericana de Psicología: Ciencia y Tecnología. (1): 61-71. Recuperado de: https://dialnet.unirioja.es/descarga/articulo/4905155.pdf

GOLDSTEIN, A.P.; SPRAFKIN, R.P.; GERSHAW, N.L. Y KLEIN, P. (1989). Habilidades sociales y autocontrol en la adolescencia. Barcelona: Martínez Roca.

HIDALGO, C.G. y ABARCA, N. (1990). Desarrollo de las HHSS en estudiantes universitarios. Bogotá, Colombia. Revista Latinoamericana de Psicología. 22 (2): 265 282.

IRUARRIZAGA, I., GÓMEZ-SEGURA, J., CRIADO, T., ZUAZO M. \& E. SASTRE (1997). Reducción de la ansiedad a través del entrenamiento en habilidades sociales. Revista Electrónica Motivación y Emoción. Volumen: 2 Número: 1. Recuperado de http://reme.uji. es/articulo/airuai462031198/texti,html.

KOLAKOSKI, L. (1988). La filosofía positivista. Madrid: Cátedra.

LISETTE-SEGURA, G. y LÓPEZ, P. (2014). El aprendizaje de las HHSS en la universidad. análisis de una experiencia formativa en los grados de educación social y trabajo social. Formación Universitaria. La Serena, Chile. 7(4): 1-15. Recuperado de: www.scielo.cl/scielo.php?pid=S0718-0062014000400004\&script=sci_arttext

MEICHENBAUM, D., BUTLER, L. Y GRUSON, L.(1981). TOWARD A CONCEPTUAL MODEL OF SOCIAL COMPETENCE. En J. Wine y M. Smye (Comps.), Social competence. Nueva York: Guilford Press

MICHELSON, L. Y WOOD, R. (1980), Development and Psychometric properties of the Children's Assertive Behavior Scale. Journal of Behavioral Assessment, 4, 3-14

MIRANDA - ZAPATA, E.; RIQUELME - MELLA, E.; CIFUENTES - CID, H. y RIQUELME - BRAVO, P. (2014). Análisis factorial confirmatorio de la escala de HHSS en universitarios chilenos. Revista Latinoamericana de Psicología. Recuperado de: http://www.redalyc.org/articulo.oa?id=80532608001

MONJAS, M. (2007). Cómo promover la convivencia: programa de asertividad y habilidades sociales (PAHHSS). España: Editorial CEPE.

MORÁN, V. y OLAZ, F. (2014). Instrumentos de evaluación de HHSS en América Latína. Revista de Psicología. Recuperado de: www.revistapsicologia.uchile.cl/index.php/RDP/article/ download/32877/34633

ORDAZ - HERNÁNDEZ, M. (2013). La educación de HHSS desde la extensión universitaria. Propuesta de acciones. Cuba. Recuperado de: www.scielo.br/pdf/er/n50/n50a17.pdf 
RISO, W. (1988). Entrenamiento asertivo: aspectos conceptuales, evaluativos y de intervención. Medellín: Ediciones Rayuela

RODRÍGUEZ-MACAYO, E., VIDAL-ESPINOZA, R. (2018). Nivel de desarrollo de las HHSS en estudiantes de la facultad de educación cohorte de ingreso 2018, de la Universidad Católica Silva Henríquez. Proyecto Fondo Interno Documento inédito no publicado de la UCSH.

RUÍZ-ARIAS, V. y JARAMILLO-CAMPILLO, E. (2010). Habilidades sociales en estudiantes de psicología de la Universidad Cooperativa de Colombia. Sede Colombia. Colombia.

https://revistas.ucc.edu.co/index.php/pe/article/download/366/370

SÁNCHEZ - TERUEL, D.; ROBLES - BELLO, M.A. y GONZÁLEZ - CABRERA. M. (2015). Competencias sociales en estudiantes universitarios de ciencias de la salud. Educación Médica. Recuperado de: https://www.google.cl/search?q=Educaci $\% \mathrm{C} 3 \% \mathrm{~B} 3 \mathrm{n}+\mathrm{M} \% \mathrm{C} 3 \% \mathrm{~A} 9 \mathrm{dica}+2015 \% 3 \mathrm{~B} 16 \% 3$ $\underline{\mathrm{A} 126-30}$ 